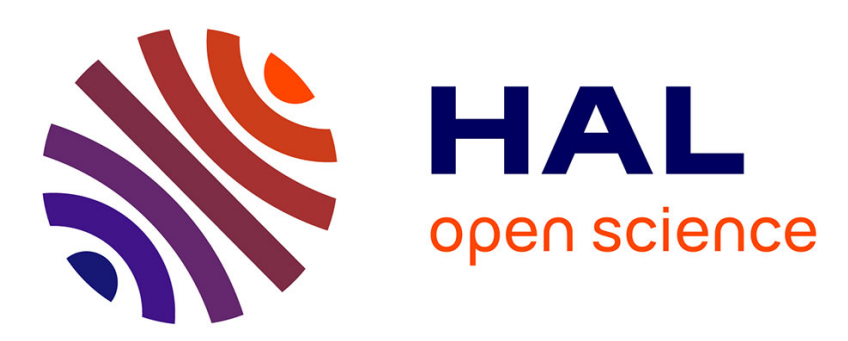

\title{
La catégorisation au cour de l'activité avec des tout-petits Manières de dire et manières de faire le travail dans les crèches
}

Sophie Odena, Anne-Lise Ulmann

\section{- To cite this version:}

Sophie Odena, Anne-Lise Ulmann. La catégorisation au cœur de l'activité avec des tout-petits Manières de dire et manières de faire le travail dans les crèches. Revue des politiques sociales et familiales, 2020, Socialisation de enfants. Intentions, attentions et injonctions, 136-137, pp.24-37. 10.3406/caf.2020.3432 . hal-03169887

\section{HAL Id: hal-03169887 \\ https://hal.science/hal-03169887}

Submitted on 14 May 2021

HAL is a multi-disciplinary open access archive for the deposit and dissemination of scientific research documents, whether they are published or not. The documents may come from teaching and research institutions in France or abroad, or from public or private research centers.
L'archive ouverte pluridisciplinaire HAL, est destinée au dépôt et à la diffusion de documents scientifiques de niveau recherche, publiés ou non, émanant des établissements d'enseignement et de recherche français ou étrangers, des laboratoires publics ou privés. 


\section{La catégorisation au cœur de l'activité avec des tout-petits}

Manières de dire et manières de faire le travail dans les crèches

Anne-Lise Ulmann

Sophie Odena

\author{
Maître de conférences, habilitée à diriger les recherches (Hdr), Conservatoire national \\ des arts et métiers (Cnam) - membre du Centre de recherche sur le travail \\ et le développement (Crtd) Ea 4132. \\ Chercheuse associée au Laboratoire d'économie et de sociologie du travail (Lest) - \\ Centre national de la recherche scientifique (Cnrs), Aix-Marseille Université - \\ membre du Centre de recherche sur le travail et le développement.
}

\section{La catégorisation au cœur de l'activité avec des tout-petits}

Manières de lire et manières de faire le travail dans les crèches

Cet article présente la manière dont les professionnelles exerçant en crèche ont recours à des catégories pour appréhender ou caractériser individuellement et collectivement les enfants dont elles s'occupent, ainsi que les collègues avec qui elles travaillent. À travers l'exemple des repas, l'analyse met en évidence différents usages de ces catégories par les professionnelles pour ajuster la charge de travail, jauger les compétences des collègues et répondre aux nombreuses attentes des parents. Trois d'entre elles, liées au rythme de fréquentation de la crèche, donnent à voir le rapport que les professionnelles construisent aux enfants ; elles amènent à souligner l'importance des régulations collectives pour éviter que le processus de catégorisation ne devienne pas un processus de stigmatisation. En révélant leurs dimensions fonctionnelles pour l'action, ces catégories permettent de saisir les intrications des différentes dimensions du travail, depuis les tâches à faire jusqu'aux enjeux sociaux de l'accueil des enfants.

Mots-clés : Crèche - Catégorie - Professionnalité - Travail - Langage.

\section{Categorization at the core of activity with toddlers}

Ways of reading and ways of working in daycare centers

This article presents the ways in which professionals working in daycare centers resort to categories to address or characterize, individually or collectively, the children in their keeping, and their work colleagues. Through the example of meals, this analysis highlights these categories different uses by professionals to adjust workload, assess their colleagues' skills, and respond to the many expectations of parents. Three of them, linked to the nursery attendance schedules, display the relationship that the professionals build with the children. They serve to underline the importance of collective regulations to prevent categorization turning into a process of stigmatization. By revealing their functional dimensions for action, these categories bring to light meshing and merging of the various dimensions of this work, from tasks to be executed to the social implications of childminding.

Keywords: Nursery - Category - Professionality - Work - Language. 
L'accueil des jeunes enfants est soumis à des normes, une réglementation, I'obtention d'un agrément et répond à des objectifs variables selon l'évolution et les besoins de la société. Successivement outils au service de la démographie, puis de la conciliation vie familialevie professionnelle (Neyrand, 2011 ; Odena, 2019), les structures d'accueil collectif des jeunes enfants sont aujourd'hui investies d'une mission éducative et sociale qui embrasse un nombre croissant d'exigences (Giampino, 2016; Hcfea, 2019) : I'éveil de l'enfant, le soutien à la parentalité, la prise en charge de la pauvreté, des handicaps, le respect des choix éducatifs et religieux des parents... Dans ce contexte, comment les professionnelles ${ }^{1}$ s'ajustent-elles à la diversité de ces demandes sociales et politiques? Quels savoirs mobilisent-elles pour répondre à cette diversité de problématiques?

Un certain nombre de recherches ont déjà montré que les pratiques de travail de ces professionnelles étaient mal connues ou se révélaient parfois très différentes des représentations qui structurent les programmes de formation préparant à tenir ces fonctions (Cresson, 1998 ; Cartier et al., 2014 ; Ulmann et al., 2015 ; Odena, 2009). La recherche sur laquelle cet article s'appuie fait I'hypothèse que cette méconnaissance du travail avec des toutpetits tient pour une large part à la difficulté de saisir le déroulement de l'action en train de se faire du point de vue des acteurs qui la font. Les établissements d'accueil du jeune enfant (Eaje) ne sont pas aisément accessibles à des observateurs extérieurs (Ulmann et al., 2011) et quand ils le sont, la proximité du travail avec celui de la sphère domestique, caractéristique des métiers du « care » (soin aux enfants), rend délicate la manière d'appréhender l'effectivité et le sens des pratiques mises en œuvre. L'évidence de l'action peut abuser l'observateur qui peine à " apprendre quelque chose sur la façon dont les structures des activités quotidiennes sont produites et soutenues de manière ordinaire et routinisée » (Garfinkel, 2007 , p. 100). La focale d'analyse attentive aux paroles dites en situation permet de repérer des processus de catégorisation construits par les professionnelles à des fins pragmatiques pour interagir entre elles, avec les enfants et les parents. Appréhender l'action par ce prisme contribue ainsi à saisir les conceptions du travail qui structurent les manières $d^{\prime}$ agir de ces professionnelles.
Après la présentation du cadre d'analyse explicitant le processus de catégorisation et ses rapports avec la professionnalité ${ }^{2}$ les différents usages sont ici étudiés. En premier lieu, la catégorisation constitue un moyen pour garder prise avec les complexités du travail. L'activité ordinaire mais intense des repas conduit à constater l'importance de ce processus pour ajuster la charge de travail, jauger les compétences des collègues et répondre aux nombreuses attentes des parents. L'analyse se poursuivra avec trois catégories, "périsco », " occasionnel » et « régulier», créées par les professionnelles pour identifier les enfants en fonction de leur rythme de fréquentation de la crèche. Les usages de ces créations lexicales permettent de comprendre les tensions évoquées par les professionnelles pour ajuster la prise en charge des enfants à des contextes divers, notamment ceux liés à l'évolution des politiques publiques. En conclusion, ce processus de catégorisation conduit à questionner la manière dont la formation prépare à ces évolutions et met en évidence l'importance de l'échange et du travail d'équipe pour faire face aux complexités méconnues du travail avec des tout-petits.

\section{Catégoriser : une ressource pour l'action}

Contrairement à une acception fréquente mais réductrice du terme qui assimile catégorisation à stigmatisation, il est ici envisagé comme un processus mental ordinaire, nécessaire pour développer une compréhension des situations et agir sur elles. Catégoriser « consiste en une construction sélective qui restreint parmi le monde extérieur un nombre limité d'éléments »(Goffman, 1973, p. 11), et permet d'ordonner et d'établir des normes (Houdé, 1992) voire de hiérarchiser ses actions. Quel que soit son domaine, un professionnel est amené à catégoriser ; avec l'expérience, cela contribue à développer une "vision professionnelle »(Goodwin, 1994) qui lui donne de l'aisance pour traiter une grande diversité de situations, mêmes si elles sont inédites. Guy Jobert qualifie de "professionnel " "celui qui a l'œil, qui voit tout de suite que cela ne va pas » (2019, p. 23) tandis que le débutant hésite et tâtonne et, pour reprendre Daniel Arasse, « $n^{\prime} y$ voit rien » (Arasse, 2003).

De ce point de vue, le processus de catégorisation s'apparente tout d'abord à l'élaboration de repères cognitifs

\footnotetext{
${ }^{1}$ Dans la mesure où, pour cette recherche, peu d'hommes exerçant la profession ont pu être rencontrés, le féminin sera utilisé dans l'article pour faire référence aux professionnelles avec lesquelles l'équipe a travaillé.

${ }^{2}$ En référence au chercheur Jean-Marie Barbier, dans ce texte, la professionnalité sera considérée dans un sens large, à savoir : « la désignation des compétences correspondant à un exercice professionnel » (2011, p. 106).
} 
que le professionnel façonne pour lui-même, de manière à garder une certaine maîtrise de l'environnement de travail sur lequel il est censé agir. La catégorisation opère donc comme ressource pour orienter l'attention, soutenir la concentration afin de mieux guider l'action et soulager la charge cognitive lorsque l'environnement est particulièrement touffu ou sollicitant. En second lieu, la catégorisation s'articule étroitement avec la professionnalité. Quand «les acteurs procèdent par "typification", ils appréhendent la réalité à travers des catégories générales qui orientent leurs interprétations et leurs attentes »(Lacoste, 1999, p. 9). Ces repères cognitifs individuels, construits dans et pour I'action, sont aussi partagés et relient chaque professionnel aux autres personnes du métier, souvent à partir de connaissances apprises et de savoirs d'action partagés, éprouvés en situation. Ils font lien entre les professionnels et, à ce titre, relèvent de " l'instance interpersonnelle » du métier (Clot, 2007) ${ }^{3}$, contribuant à façonner la langue du métier. Enfin, la catégorisation permet d'alléger la charge subjective. En effet, la pensée pratique se satisfait souvent de « savoirs approximatifs, seulement partiellement clairs [parfois même] incohérents ou contradictoires »(Schutz, 1987, p. 220), mais qui, dans tous les cas, restent suffisants pour agir.

Accéder aux catégories forgées par les professionnels dans le cours de l'action donne accès à la manière dont se forgent des représentations au sein de groupes sociaux confrontés aux mêmes activités. En effet, les catégories n'existent pas en tant que telles; elles sont des constructions qui se donnent à voir comme des points de vue sur le monde qui contribuent à faire advenir la réalité qu'elles évoquent (Bourdieu, 1993).

Qu'en est-il des catégorisations faites par les professionnelles de la petite enfance : comment sélectionnent-elles des informations pour agir avec les enfants? Quels savoirs, valeurs ou encore conceptions éducatives interfèrent-ils dans leurs choix ? Comment ces catégories peuvent-elles aider à conduire le travail dans les moments plus intenses? Pour répondre à ces questions, l'attention s'est portée sur le langage. C'est en effet par la parole que les professionnelles se manifestent et font connaître le sens de ce qu'elles font. Ce langage est "lui-même situé, indexical renvoyant sans cesse au contexte dont il dépend et dont il se nourrit » (Lacoste, 1999, p. 10). Rester attentif à la manière dont les professionnelles peuvent parfois qualifier un groupe de filles de "petites Barbies », certains parents de "pénibles » ou évoquer ainsi le comportement d'un enfant - « même s'il est passé chez les moyens c'est encore un petit »-montre qu'elles mobilisent des savoirs sociaux véhiculés par le langage qui guident certains de leurs comportements. S'y révèle l'intrication entre les composantes cognitives et normatives de l'action, entre les valeurs et les savoirs, qui organise la connaissance ordinaire qui permet d'agir en situation (ibid.).

Pour appréhender ces processus de catégorisation, une approche d'orientation sociolinguistique a été choisie (Lacoste, 1999 ; Borzeix et Fraenkel, 2001) permettant de relever les manières de parler de ces professionnelles. En portant l'attention sur l'interaction verbale, au sens d'Erving Goffman - "l'ensemble de l'interaction qui se produit en une occasion quelconque quand les membres d'un ensemble donné se trouvent en présence continue les uns des autres »(Goffman, 1973, p. 23), ces usages langagiers ont été saisis pour tenter de comprendre ce qu'ils cherchaient à désigner. Qu'est-ce, pour la professionnelle qui s'exprime mais aussi pour ses collègues, qu'une "petite Barbie », un " parent pénible », ou encore un «moyen »? Quels savoirs et connaissances fondent ces désignations ? Ces dernières sont-elles seulement liées aux situations où elles ont été entendues? Ce premier angle d'analyse est enrichi par la prise en compte plus phénoménologique des activités effectuées pour se saisir de ce que ces professionnelles font dans les situations où elles sont engagées (encadré 1).

Pour parvenir à la compréhension de ces situations, deux focales d'observation ont été combinées : une première axée sur le fonctionnement global et les interactions des professionnelles avec les enfants et entre elles, et une deuxième, plus resserrée sur des situations ciblées. Les premières observations ont permis de saisir les manières d'agir individuellement et collectivement, les lexiques professionnels et les ajustements d'organisation au sein des équipes. Différents processus de nomination ont alors été observés, analysés plus précisément dans un second temps lors de l'étude de situations telles que les repas, les sorties, I'arrivée et le départ des enfants. Ainsi, I'attention aux interactions verbales, non verbales, aux jeux de regards est venue éclairer les manières de faire, de penser, de modifier et de préparer I'action. Elle a permis de comprendre comment s'intriquent ou s'hybrident des prescriptions, des convictions, des savoirs et des rapports sociaux dans la conduite du travail avec les tout-petits.

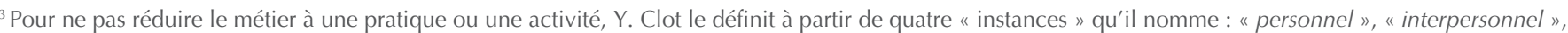
« transpersonnel » et « impersonnel ». Ces instances sont dans un rapport de tension créative.
} 


\section{Méthodologie}

La recherche réalisée sur vingt mois entre 2017 et 2019 a été conduite avec une équipe pluridisciplinaire, composée de chercheurs en sciences de la formation, sociologues et de professionnels des métiers du social1. Elle s'est déroulée, pour la partie présentée dans cet article, dans quatre établissements d'accueil du jeune enfant (Eaje), sur deux territoires, Provence-Alpes-Côte d'Azur et île-de-France. Ces derniers ont été choisis en fonction de la localisation des équipes de recherche, de leur connaissance déjà acquise avec d'autres recherches de ces milieux professionnels et des possibilités laissées aux chercheurs d'y effectuer des observations de manière récurrente et régulière (tableau, p. 28).

La recherche porte sur des professionnelles qualifiées : éducatrices de jeunes enfants (Eje), auxiliaires de puériculture (Aux), certificats d'aptitude professionnelle (Cap) Petite enfance, toutes volontaires et intéressées pour participer.

L'étude a été menée avec une méthodologie d'inspiration ethnographique qui peut être spécifiée par trois principes :

- une immersion au sein de chaque structure en adaptant tout d'abord le régime de présence de l'équipe de recherche aux souhaits des professionnelles, puis, progressivement, en venant plus librement. Cette présence régulière (deux journées par semaine en moyenne sur les neuf mois de la première année, et une journée par quinzaine pendant cinq mois la seconde année) a permis de gagner la confiance des professionnelles, qui intégraient les enquêteurs dans leur univers de travail en les faisant participer très largement à la vie de la structure ;

- la combinaison de deux registres d'observation : celui d'une extériorité du chercheur ne participant pas à l'action puis, avec le temps, celui d'une observation impliquée limitée à des aides ponctuelles dans des moments denses (surveiller des enfants, répondre à la sollicitation directe d'enfants, aider à quelques tâches matérielles (débarrassage des tables, rangements...) ;

- des entretiens sur ou à propos du travail, enregistrés lorsque les conditions le permettaient, qui s'apparentent plutôt au principe de la « conversation » de manière à réduire " l'artificialité de la situation d'entretien et l'imposition par l'enquêteur de normes méta-communicationnelles perturbantes $»^{2}$. À ces entretiens, des restitutions régulières des résultats intermédiaires ont été ajoutées (tableau 1) avec l'ensemble des personnels disponibles aux dates proposées, engendrant souvent la conduite d'autres observations. Cette "récursivité » des entretiens et des échanges « qui permet de s'appuyer sur ce qui a été dit pour produire de nouvelles questions ${ }^{3}$, explique que chaque structure a donné lieu à des investigations spécifiques, même si la démarche méthodologique a été la même.

Les analyses ont été conduites au sein de chaque Eaje et ont ensuite été comparées entre elles de manière à identifier des constantes dans les manières de nommer les enfants ou d'agir avec eux et des différences sur le fonctionnement de ces établissements et les manières d'appréhender les enfants. Les comparaisons ont permis une certaine triangulation des données permettant de " croiser les informateurs pour ne pas être prisonnier d'une seule source » ou de "faire varier les informateurs en fonction de leur rapport au problème traité »4. L'objectif du recueil de ces données empiriques est de rendre compte des multiples registres du réel social pour s'acheminer vers une connaissance des spécificités de ce travail. Ce point de vue n'est pas construit en extériorité mais avec et à partir des acteurs qui font le travail, au risque parfois de questionner les catégories des chercheurs.

L'approche ethnographique, parce qu'elle présente des faits vécus comme des expériences individuelles ou collectives, ne se limite pas à des constats de type microsociologique. Elle peut, même si c'est de manière plus limitée que d'autres approches visant la représentativité, montrer comme ces petits faits " présentent des propriétés qui expriment leur appartenance à l'ordre social et qui relèvent de leur enchâssement dans ces mécanismes [...]. L'originalité de la situation intellectuelle de l'ethnographie tient précisément à la capacité dont elle dispose de circuler entre propriétés situationnelles et propriétés structurelles du fait social et de les éclairer les uns par les autres. ${ }^{5}$

${ }^{1}$ Cette recherche a également concerné l'usage des catégories chez des assistantes maternelles faisant intervenir une troisième équipe de recherche, celle d'AgroSup (Institut national supérieur de sciences agronomiques de I'alimentation et de l'environnement) de Dijon avec Paul Olry, professeur en sciences de l'éducation, Chantal Revaillot et Cécile Lagarde, de l'Institut régional supérieur du travail éducatif et social de Bourgogne et associées à Agrosup Dijon. Bien que l'activité des assistantes maternelles ne soit pas présentée dans cet article, les données recueillies sur l'ensemble des terrains ont été analysées collectivement. Les résultats présentés sont aussi le produit du travail conduit avec cette équipe.

${ }^{2}$ Olivier de Sardan J.-P., 1995, La politique de terrain. Sur la production des données en anthropologie, Enquête - Les terrains de l'enquête. Voir le site https://sspsd.u-strasbg.fr/IMG/pdf/Olivier_de_Sardan_1995_enquete-263-1-la-politique-du-terrain.pdf (consulté le 16 décembre 2019 ), p. 83.

3 lbid., p. 91.

${ }^{4}$ Ibid., p. 94.

${ }^{5}$ Schwartz O., 2011[1993], L'empirisme irréductible. La fin de l'empirisme?, , Anderson N. (dir.), Le Hobo, sociologie du sans-abri, Paris, Armand Colin, p. 336-380.

\section{Catégoriser : un moyen pour garder prise sur les complexités du travail}

L'immersion au sein d'une crèche permet très vite de repérer que certains moments de la journée sont particulièrement denses et délicats à conduire pour les professionnelles. Les repas des enfants, des plus petits comme des plus grands, donnent à voir un réglage subtil qui incombe le plus souvent aux auxiliaires de puéricultures et aux professionnelles titulaires du certificat d'aptitude professionnel (Cap), plus rarement aux Eje, à l'exception d'une crèche associative parentale observée où la structure fait le choix de ne pas embaucher d'auxiliaires dans ses effectifs (tableau p. 28). Cette division du travail, cohérente avec la partition du médical et de l'éducatif (Rayna, 2003 ; Odena, 2009 ; Ulmann, 2015), peut parfois faire écho à une relégation de "sale boulot " dans le travail de care (" soins") (Le Floch, 2008) vers les personnes les moins formées, tant ce moment se révèle peu gratifiant et éprouvant psychiquement et physiquement.

Revue des politiques sociales et familiales $n^{\circ} 136-137-3^{\text {e }}$ et $4^{\text {e }}$ trimestres 2020 


\begin{tabular}{|c|c|c|c|c|}
\hline \multirow[b]{3}{*}{$\begin{array}{l}\text { Enfants } \\
\text { accueillis }\end{array}$} & \multicolumn{2}{|c|}{ Région Provence-Alpes-Côte d'Azur } & \multicolumn{2}{|c|}{ Région île-de-France } \\
\hline & Associatif & Municipal & Associatif-Parental & Municipal \\
\hline & $\begin{array}{l}45 \text { places. } \\
\text { Accueil concernant } \\
63 \text { enfants. }\end{array}$ & $\begin{array}{l}30 \text { places. } \\
\text { Accueil concernant } \\
40 \text { enfants. }\end{array}$ & $\begin{array}{l}16 \text { enfants à partir de } 10 \text { mois. } \\
\text { Accueil concernant } \\
18 \text { enfants. }\end{array}$ & $\begin{array}{l}50 \text { enfants. } \\
\text { Accueil concernant } \\
55 \text { enfants dont } 4 \text { porteurs } \\
\text { de handicap. }\end{array}$ \\
\hline Personnel & $\begin{array}{l}1 \text { puéricultrice (direction). } \\
2 \text { Eje (dont une en } \\
\text { codirection). } \\
6 \text { auxiliaires. } \\
6 \text { animatrices d'éveil (Cap). } \\
1 \text { cuisinière. } \\
2 \text { agents d'entretien. }\end{array}$ & $\begin{array}{l}1 \text { infirmière (direction). } \\
1 \text { Eje (codirection). } \\
4 \text { auxiliaires. } \\
3 \text { agents d'animation (Cap). } \\
1 \text { apprentie } \\
\text { Cap Petite enfance. } \\
1 \text { stagiaire Eje. }\end{array}$ & $\begin{array}{l}4 \text { Eje, dont une en fonction } \\
\text { de direction à mi-temps. } \\
2 \text { apprenties Cap } \\
\text { et baccalauréat professionnel. } \\
1 \text { cuisinière. } \\
\text { Présence quotidienne } \\
\text { d'un parent par demi-journée. }\end{array}$ & $\begin{array}{l}4 \text { Eje dont une en direction } \\
\text { adjointe. } \\
9 \text { auxiliaires }+1 \text { liée à la } \\
\text { situation de certains enfants. } \\
1 \text { Cap pour les fonctions } \\
\text { logistiques. } \\
2 \text { apprentis (Aux et Eje). }\end{array}$ \\
\hline $\begin{array}{l}\text { Personnel } \\
\text { observé }\end{array}$ & $\begin{array}{l}2 \text { Eje. } \\
4 \text { auxiliaires. } \\
4 \text { animatrices d'éveil. }\end{array}$ & $\begin{array}{l}1 \text { Eje }+1 \text { Eje stagiaire. } \\
3 \text { auxiliaires. } \\
2 \text { agents d'animation. } \\
1 \text { Infirmière. }\end{array}$ & $\begin{array}{l}2 \text { Eje. } \\
1 \text { Cap (apprentie). }\end{array}$ & $\begin{array}{l}1 \text { Eje. } \\
5 \text { Aux. }\end{array}$ \\
\hline Organisation & $\begin{array}{l}3 \text { sections : } \\
\text { - bébés } \\
\text { - moyens } \\
\text { - grands }\end{array}$ & $\begin{array}{l}\text { Deux groupes : un groupe } \\
\text { de bébés ( } 0-1 \text { an) et un groupe } \\
\text { de moyens-grands. }\end{array}$ & $\begin{array}{l}\text { Deux grands espaces de jeux: } \\
\text { un pour les plus jeunes avant } \\
\text { la marche, un autre pour } \\
\text { après la marche. } \\
\text { Principe de la référence } \\
\text { pour les enfants. }\end{array}$ & $\begin{array}{l}\text { Organisation en deux groupes } \\
\text { d'âge : deux groupes de } \\
\text { bébés de } 2 \text { ans et un groupe } \\
\text { de } 2-3 \text { ans). }\end{array}$ \\
\hline $\begin{array}{l}\text { Spécificité du } \\
\text { fonctionnement }\end{array}$ & $\begin{array}{l}\text { Crèche au public très } \\
\text { hétérogène. } \\
\text { Partenariat avec un centre } \\
\text { d'accueil mère-enfant } \\
\text { (certaines places sont } \\
\text { réservées). }\end{array}$ & $\begin{array}{l}\text { Seulement deux sections : les } \\
\text { bébés et les moyens-grands. }\end{array}$ & $\begin{array}{l}\text { Les parents présents ne } \\
\text { s'occupent que de leur } \\
\text { enfant et participent aux } \\
\text { tâches matérielles (courses, } \\
\text { nettoyage...). } \\
\text { Se réfère à la pédagogie } \\
\text { Pickler Loczy. }\end{array}$ & $\begin{array}{l}\text { Beaucoup d'activités en âges } \\
\text { mélangés. } \\
\text { Professionnelles affectées } \\
\text { sur un groupe mais menant } \\
\text { des activités avec tous les } \\
\text { enfants : tableau d'affectation } \\
\text { d'activités pour tous les } \\
\text { moments de la journée. }\end{array}$ \\
\hline $\begin{array}{l}\text { Ergonomie } \\
\text { des espaces }\end{array}$ & $\begin{array}{l}\text { Crèche sur trois étages avec } \\
\text { une petite cour intérieure. }\end{array}$ & $\begin{array}{l}\text { Locaux spacieux pour les } \\
\text { moyens grands et petit espace } \\
\text { pour les bébés. } \\
\text { Grand jardin. }\end{array}$ & $\begin{array}{l}\text { Locaux spacieux. } \\
\text { Espace dédié pour les repas } \\
\text { et pour les temps de repos. }\end{array}$ & $\begin{array}{l}\text { Eaje scindé sur deux espaces, } \\
\text { communiquant par un } \\
\text { escalier. } \\
\text { Jardin intérieur. } \\
\text { Repas organisés au sein } \\
\text { de l'espace de vie de chaque } \\
\text { groupe d'enfants. }\end{array}$ \\
\hline $\begin{array}{l}\text { Restitutions } \\
\text { organisées }\end{array}$ & $\begin{array}{l}\text { Une réunion de démarrage } \\
\text { en présence de l'ensemble } \\
\text { des équipes. } \\
\text { Une restitution intermédiaire. } \\
\text { Une réunion croisée. }\end{array}$ & $\begin{array}{l}\text { Trois restitutions des résultats } \\
\text { intermédiaires intégrant } \\
\text { l'ensemble de l'équipe } \\
\text { et les parents employeurs. }\end{array}$ & $\begin{array}{l}\text { Quatre réunions } \\
\text { de restitutions des résultats } \\
\text { permettant d'échanger } \\
\text { avec l'ensemble de l'équipe. }\end{array}$ & Deux réunions de restitution. \\
\hline
\end{tabular}

Eje : éducatrices de jeunes enfants ; Cap : certificat d'aptitude professionnelle ; Aux : auxiliaire de puériculture ; Eaje : établissement d'accueil du jeune enfant.

\section{Le repas : un moment complexe à organiser}

Le repas dans un Eaje concentre un ensemble d'exigences techniques et éducatives. En premier lieu, la contenance des enfants qui sont fatigués en fin de matinée et le temps contraint pour ne pas empiéter sur un éventuel deuxième service, sur le temps de la sieste ou sur le planning des personnels de service génère une pression temporelle. S'y ajoutent les nombreuses manipulations requérant une certaine dextérité pour faire laver les mains des enfants, les installer à table avec leur serviette, dresser le couvert, servir, couper les aliments, vérifier leur tempé- rature, éplucher les fruits, ou encore aider à manger. Ces manipulations effectuées dans un espace souvent petit (tableau) ne sont pas sans entraîner des débordements. On entend fréquemment les professionnelles se fâcher, voire crier : " non je ne te redonne pas d'eau, tu ne bois pas, tu t'amuses. Tu as vu, tout s'est renversé... Ça suffit Mattias ${ }^{4}$, tu arrêtes maintenant! » [auxiliaire de puériculture (Aux.), crèche municipale]; "Oh là là ! Ce n'est pas possible aujourd'hui ! Qu'est-ce qui se passe? C'est quoi ça ? Reposez ces assiettes! " [certificat d'aptitude professionnelle $(\mathrm{Cap})^{5}$, crèche associative $\left.{ }^{6}\right]$. Enfin, $c^{\prime}$ est 
aussi un temps éducatif pour faire découvrir les noms des plats, apprendre à manger de manière autonome, attendre son tour pour le service, demander poliment... Cette dernière exigence est très variable en fonction du planning et de l'organisation au sein de la structure. Parfois, les professionnelles échangent avec les enfants sur la nourriture : "Qui connaît ce que c'est? Des carottes! Non, regarde ce n'est pas la couleur... les carottes c'est orange et là... c'est de quelle couleur... ? Rouge ! Très bien Philae, alors si c'est rouge c'est... c'est du chou rouge " (Aux., crèche associative) - mais le plus souvent, le plat est juste nommé. Cette dimension éducative consiste surtout à apprendre aux enfants à maîtriser leur impatience : "Elsa, je t'ai vue, j'ai compris que tu avais faim, mais là, tu vois, je sers Ptolémée... Chacun son tour. Ça n'ira pas plus vite, même si tu cries... tu attends ton tour, comme les copains. Va t'asseoir, s'il te plaît. » (Aux., crèche municipale).

Ces verbatim fréquemment entendus ne rendent cependant pas bien compte de la fatigue éprouvée, des marques de colère retenues quand de la nourriture a taché un vêtement, «Bon, je n'ai plus qu'à me changer! », voire des remarques glissées discrètement à la collègue près du chariot des plats sur les difficultés supplémentaires qu'il va falloir maîtriser : «Pshhh... c'est des petits pois ! Ça va encore être galère... » (Aux., crèche municipale). Tout cet ensemble de bruits, remarques, cris, soupirs créent une atmosphère plus ou moins pesante en fonction des établissements.

Dans la crèche associative parentale (Ap), le repas paraît fortement en contraste avec les autres Eaje observés. D'une part, le bruit est nettement moindre, en lien avec le nombre plus réduit d'enfants. D'autre part, les Eje déjeunent en même temps que les enfants par petits groupes. Enfin, la cuisinière est présente dans la salle lors du déjeuner, même si elle reste en retrait. La plupart du temps, debout derrière un comptoir qui fait office de passe-plats, elle observe le déroulement du déjeuner, échangeant parfois avec les Eje ses impressions sur le comportement des enfants, qu'elle connaît par leur prénom. "Maxime, je trouve qu'il change en ce moment, il se débrouille bien maintenant... ». Les parents de perma- nence préparent le couvert des tables, mais déjeunent un peu à l'écart avec leur propre enfant sur une petite table conçue pour deux et ne participent pas, du moins lors de nos présences, à ces échanges. Ce partage du repas avec les enfants mais aussi avec la cuisinière donne une tonalité conviviale à ce moment. Il ne s'agit plus seulement du repas des enfants mais d'un repas partagé avec les professionnelles. La nature des échanges avec les enfants s'en ressent, parce qu'ils ne sont plus seulement à tonalité éducative, centrés sur eux, mais relèvent davantage de l'échange ordinaire avec une personne tierce (la cuisinière) : "Tu as mis de la cannelle? C'est super bon! Vous êtes d'accord les enfants ? Félicitez Eva alors, dites-lui que vous aimez » (Eje, crèche Ap). Ces échanges ouverts sur la vie quotidienne tendent à décrisper le moment du repas, qui paraît se dérouler plus facilement. En se décalant d'une relation strictement éducative, ces interactions avec les enfants semblent paradoxalement contribuer davantage à l'apprentissage de la sociabilité.

\section{Apprécier les capacités des enfants et répartir équitablement la charge de travail}

Dans les échanges avec les directrices de ces établissements, l'organisation des repas est fréquemment évoquée comme une préoccupation qui conduit à des ajustements, des "tâtonnements », pour reprendre le terme de I'une d'elles, compte tenu d'un ensemble de contraintes (l'espace, les qualifications des personnels, les temps de pause, le nombre d'enfants d'une même classe d'âge, etc.). "Avant d'en arriver à faire comme maintenant, expliquera l'Eje responsable de la crèche parentale, nous avons beaucoup travaillé et cherché ». Dans les autres Eaje, ces «tâtonnements » soit font l'objet d'un travail collectif au sein des équipes, soit restent la prérogative de la directrice avec son adjointe. Les échanges en réunion à propos des repas (quand ils existent) portent moins sur les dimensions éducatives de ce moment que sur la répartition équitable des enfants entre les professionnelles. Cette répartition s'établit à partir d'une attribution de capacités portée sur chacun d'eux : Ptolémée, « qui n'est pas autonome pour manger » est, par exemple, affecté dans un sous-groupe avec moins d'enfants à aider pour

\footnotetext{
${ }^{4}$ Les prénoms des enfants et des professionnelles cités ont été modifiés afin de préserver leur anonymat.

${ }^{5}$ Plusieurs termes désignent les titulaires du Cap petite enfance en Eaje : agent, animatrice d'éveil, agent territorial spécialisé des écoles maternelles (et dans certaines crèches municipales). Dans la recherche, cette catégorie de professionnel est, comme les Eje ou les auxiliaires de puériculture, en interaction avec les enfants durant tous les moments de la journée. Par souci d’unité, le choix a été d'utiliser dans cet article le sigle Cap en référence à leur formation commune dans les citations de verbatim.

${ }^{6}$ Le choix a été fait de préciser après les analyses concernant une structure ou un verbatim le statut des crèches concernées (associatif, municipal, parental), celui-ci pouvant, dans certains cas, impacter le mode d'organisation et de gestion de la structure et, par conséquent, les modalités de travail, comme cela a pu être observé dans de précédentes recherches (Odena, 2009).
} 
la professionnelle. Cet équilibre subtil de la charge de travail montre que les catégories servent de décrypteurs de leurs comportements en lien avec l'activité à réaliser. Lorsque ce travail collectif sur la manière d'appréhender le comportement des enfants pour équilibrer la charge de travail entre les membres de l'équipe n'est pas systématisé au sein de l'établissement, davantage de tensions avec les enfants sont observés dans les moments plus denses de l'activité : "On l'a passé ici [chez les moyens] parce qu'il se débrouille bien pour manger, mais pour moi, il serait mieux chez les petits. [...] II n'est pas à l'aise avec les autres, il est toujours collé à nous... » (aux., crèche municipale). L'attribution de capacité de cet enfant n'étant manifestement pas partagée au sein de l'équipe, des agacements sont perceptibles au moment de sa prise en charge pour le repas.

Ces liens entre la catégorisation et la charge de travail sont patents pour les repas mais ils se constatent dans chaque situation où existe une certaine complexité, telle une sortie ou une activité ludique, qui va impliquer des ajustements de charge entre les professionnelles. " $A h$ non, mais pas celui-là ! Si tu m'envoies celui-là, tu en reprends deux! » (échange entre un agent d'animation et une auxiliaire, pour l'organisation d'une activité). Plus ou moins formalisé, ce ratio catégorie d'enfant-charge de travail vient compléter le ratio gestionnaire effectif d'enfants-effectifs de professionnels, inopérant pour maîtriser la complexité d'une situation.

Si ces catégories constituent des ressources pour équilibrer les charges de travail au sein du collectif, elles n'enferment pas pour autant l'enfant dans une catégorie (" agité », " petite princesse », " rapide», "prêt pour l'école »...). Celle-ci est établie en fonction de l'action à conduire et paraît prendre en compte de nombreux paramètres : I'activité, le nombre d'enfants et de professionnelles présents, le moment de la journée... II ne s'agit donc pas d'un processus qui stabilise, fige, voire assigne l'enfant à une désignation, mais qui relève plutôt d'une manière de l'appréhender qui s'ajuste en permanence. Dans certaines structures, qui fonctionnent en privilégiant un principe d'âges mélangés, de véritables " dynamiques de catégorisations » sont observées permettant, pour un même enfant, que ces attributions de capacités soient fréquemment rebattues. Ainsi catégorisé initialement au regard des comportements de ses parents, ( "il aime danser, mais son papa est Dj ») ou de normes comportementales liées à l'âge, l'enfant, lui-même, fait évoluer les regards portés sur lui : il change, progresse, surprend et, finalement, prend une part active dans ce processus. Ces changements des enfants qui influent sur les catégories portées sur eux semblent procurer une certaine satisfaction aux professionnelles, comme s'ils apportaient la preuve de leur travail qui ne se voit pas. Ces remarques permettent d'ajuster en conséquence les manières de les prendre en charge.

\section{Catégoriser : jauger en situation les compétences de ses collègues}

Le processus de catégorisation ne se limite pas à identifier les capacités des enfants mais concerne également la manière dont les professionnelles s'évaluent mutuellement. La catégorisation signale ici des compétences professionnelles attendues mais néanmoins implicites. "Elle se laisse déborder » dira une auxiliaire de crèche associative à propos de sa collègue, auxiliaire moins expérimentée. Cette remarque est liée au fait que cette personne prend trop de temps pour le lavage des mains des enfants et ne voit pas qu'elle met en difficulté sa collègue avec son groupe qui attend son tour.

Ces manières d'apprécier et de catégoriser des collègues ne sont pas établies sans raison mais s'effectuent dans un contexte donné. Le processus de catégorisation que structurent les professionnelles sur leurs manières d'agir varie en fonction des contraintes du moment où se déroule l'activité. Prendre du temps pour faire laver les mains des enfants n'est pas ce qui détermine la catégorisation, il faut le contexte de l'action. Ainsi, le temps au lavabo pour le lavage des mains au moment du repas n'est pas perçu pareillement lorsque ce même temps est pris après l'activité peinture. La hiérarchisation des buts diffère : dans ce dernier cas, ce qui prime, c'est d'apprendre à l'enfant à être « autonome et ne pas faire à sa place ». Cette même action dans le contexte du repas, avec ses contraintes temporelles, ne convient pas. Le but visé ici n'est plus l'autonomie de l'enfant, mais le respect d'une temporalité contrainte.

Ces jugements de compétence circulent entre les professionnelles, témoignant de la reconnaissance (ou non) qu'elles s'accordent mutuellement sur les pratiques. Ces catégorisations qui s'élaborent en combinant I'action et son contexte intègrent également l'attention aux autres professionnels. La place des autres dans la conduite de sa propre activité semble très importante, comme l'évoquent les façons de parler à propos de collègues : celle qui " chante tout le temps » ou qui « crie » est " assommante à la fin » (Aux., crèche associative) ; celle qui est «brouillonne », qui « s'agite trop » et qui, par son com-

Revue des politiques sociales et familiales $n^{\circ} 136-137-3^{e}$ et $4^{e}$ trimestres 2020

30 Socialisation des enfants - Intentions, attentions et injonctions 
portement, n'arrive pas à « ce que les enfants se posent» au risque d'entraîner des difficultés pour les autres ; celle qui n'est pas « au bon endroit » et, de ce fait, ne peut " pas maîtriser ce qui se passe »(Aux., crèche municipale).

L'explicitation de ces catégories permet d'accéder à la hiérarchisation de normes implicites, portées individuellement tout en étant partagées collectivement. Y sont décelées les conceptions de la professionnalité véhiculées par chacune et par la structure où elles interviennent. Ce qui semble distinguer la professionnelle compétente de celle qui n'est pas catégorisée comme telle par ses collègues paraît directement lié à cette capacité à réagencer, combiner, articuler des principes de travail avec des éléments contingents comme la particularité de l'instant, le contexte, la connaissance de l'enfant, le souci des collègues... Celles qui ne savent pas faire mettent en difficulté l'équipe. Cette plasticité qui articule I'activité au contexte d'action, manifestement essentielle pour la fluidité du travail collectif, ne semble pas faire l'objet d'apprentissages dans le cours des formations.

\section{Des catégories mises à mal par la diversité des demandes parentales}

Le repas des enfants n'est pas seulement complexe à tenir du point de vue de son organisation, il l'est aussi en raison de la diversité croissante des demandes parentales pouvant se porter sur la nourriture. Ce moment peut alors être analysé comme un révélateur de la pluralité des normes qui encadrent la prise en charge éducative des enfants : qu'il s'agisse de nourritures liées à la religion, d'un choix parental d'alimentation végétarienne ou végan, de demandes particulières comme des biberons donnés chauds et non froids ou préparés avec des laits végétaux et non d'animaux, les débats de normes et les arbitrages qui en découlent sont nombreux, complexes, et source d'implication pour chacun. Ils ne sont donc pas seulement techniques, ils font aussi intervenir les valeurs que chacun met dans son propre travail.

S. (Aux.) s'apprête à prendre en charge le premier jour de Vanessa, petite fille âgée de 3 mois. La directrice est présente également pour cet accueil. S. prend I'enfant dans les bras et demande à la mère, qui va partir, si Vanessa a I'habitude de prendre son biberon chaud ou froid. Expliquant qu'elle le préfère plutôt chaud, la mère prend congé. $S$ repart dans la salle de jeux avec I'enfant mais elle est interpellée par la directrice.

- Directrice : S., "j'ai déjà dit de ne pas demander aux parents ce qu'ils souhaitent sur la température des bi- berons. Vous savez bien qu'on leur donne froid. Alors, pourquoi posez-vous cette question?"

- S. : "Pour le premier jour, je pensais que c'est mieux de lui donner comme elle aime? Pourquoi c'est mieux? Elle n'a pas le droit d'avoir son goût à elle et ne pas aimer le lait froid ? [...]»

- Directrice : "Je l'ai déjà dit, on ne pose cette question aux parents que si l'on remarque que l'enfant ne veut pas boire à température ambiante. »

S. repart avec Vanessa, manifestement pas convaincue de l'argument (crèche municipale).

Pour un autre enfant dont les parents demandent la confection du biberon avec un lait végétal, la directrice du même établissement vient dans l'espace d'accueil des plus petits et explique à l'auxiliaire en charge de cet enfant : "sans prescription médicale, validée par le médecin de la crèche, je ne donne pas de laits spéciaux aux enfants. Je viens d'avoir la maman au téléphone, maintenant c'est clair. » (crèche municipale). Directement impliquée par cette décision, l'auxiliaire remet en question la légitimité de l'argument médical et fait valoir, en aparté, à sa collègue, l'argument économique et le libre arbitre des parents : "franchement, je ne vois pas en quoi ça gêne si le parent apporte les boîtes de lait... Ils ont le droit de nourrir leur enfant comme ils veulent...".

Ces sollicitations remettent en question le rapport aux règles établies. Elles interpellent notamment le savoir médical qui, jusqu'ici, s'est imposé. L'arbitrage en réponse à ces demandes se fait d'ailleurs encore en référence au champ médical, sollicité comme seule instance légitime, de manière cohérente avec I'histoire des pratiques de puériculture (Boltanski, 1969) et l'histoire des crèches. Cet arbitrage interpelle les auxiliaires lorsque leurs références aux savoirs ne sont pas médicales, mais plutôt psychologiques ou pédagogiques, et peut même être source de tensions au sein des équipes, notamment entre les auxiliaires et les Eje.

Dans une crèche associative, deux professionnelles s'interrogent sur la réponse à apporter aux parents concernant leur volonté de ne pas donner de pain à leur fille. La directrice a tranché en faveur de la même alimentation que les autres, l'enfant n'ayant pas de contre-indication médicale : "Moi, ça m'interroge quand même parce que c'est aux parents de décider de l'alimentation de leur enfant, je sais qu'ils sont sans gluten, en quoi ça nous dérange de respecter leur volonté ? Ce n'est pas tellement contraignant. » (Eje). Ce à quoi sa collègue, auxiliaire, lui avance l'argument

Revue des politiques sociales et familiales $n^{\circ} 136-137-3^{\text {e }}$ et $4^{\text {e }}$ trimestres 2020 
médical "Elle n'a pas de maladie, elle. Si on commence à accepter toutes les demandes, on n'en a pas fini ! ».

Au-delà de ces demandes parentales, semble se jouer une définition différente du rôle social de la crèche et des professionnels. L'importance croissante de la diversité des publics et des exigences parentales pose la question de la légitimité des normes mobilisées aujourd'hui pour réglementer l'accueil. Le seul argument médical, dans le cas des repas, n'est-il pas contradictoire avec la mission sociale confiée aux crèches d'accueillir une plus grande diversité de publics? Quelle place faire à la diversité de ces demandes qui peuvent se porter sur la nourriture, comme sur toute autre activité, telles que les choix de chansons, de jeux, des temps de sieste... ? Comment ces professionnelles peuventelles développer leur professionnalité si, dans ces moments délicats qui trament leur travail quotidien, elles ne prennent pas part aux débats qui permettent de les arbitrer?

\section{Catégoriser selon le rythme de fréquentation de la crèche : "régulier », "occasionnel » et " périsco »}

L'observation et l'écoute attentives des échanges entre les professionnelles a permis de faire apparaître une désignation des enfants en fonction de leur rythme de fréquentation et du type de contrat signé avec la structure. Tous les enfants ne séjournent pas à la crèche suivant les mêmes durées. Certains viennent tous les jours, d'autres quelques jours, d'autres occasionnellement quelques demi-journées, et certains sur des temps très précis comme les vacances scolaires. Ces différentes modalités de fréquentation des crèches sont liées à la mise en œuvre de la prestation de service unique (Psu) qui répond à la nécessité de rendre accessible les Eaje à de nouveaux publics (encadrés 2 et 3$)^{7}$.

Un élément a particulièrement attiré l'attention lors des immersions dans les structures. Dans la plupart des bureaux de direction (à l'exception de la crèche parentale), un tableau organisateur accroché au mur présente, selon différents codes de couleur, les catégories des enfants en fonction de leur rythme de fréquentation de la crèche. Le nom de chacun est apposé sur un carton de couleur différente selon qu'il est "régulier », " occasionnel », ou encore «périscolaire ». Ce classement visuel, en complément des outils de gestion informatique, permet d'un seul coup d'œil de gérer la présence ou l'absence des enfants, leur planning et d'avoir une représentation globale de la composition des groupes pour la journée en cours. Ces trois catégories $\mathrm{d}^{\prime}$ enfants portent-elles à conséquence sur la manière de les prendre en charge au sein de la crèche ? Comment les professionnelles les appréhendent-elles dans le cours de leurs actions avec eux?

\section{Des catégories créées conformes ou en tension avec les conceptions éducatives}

Les catégories "occasionnel, périscolaire et régulier » apparaissent comme des créations lexicales forgées par les professionnelles à partir des orientations des politiques publiques pour opérer une distinction entre les différents types de présence des enfants. Un glissement sémantique est repéré entre une catégorie issue des politiques publiques concernant les financements des établissements par la Psu vers des catégories de la pratique, qui distinguent les enfants « réguliers », « occasionnels « ou « périscolaires » dits « pé-

\section{La prestation de service unique}

Les caisses d'Allocations familiales (Caf) apportent aux établissements d'accueil du jeune enfant (Eaje) des soutiens financiers provenant du fonds national d'action sociale (Fnas). Il peut s'agir d'aides à l'investissement ou de subventions de fonctionnement. Les modalités d'attribution de ces aides sont définies nationalement par la Cnaf.

La prestation de service unique (Psu) est une aide au fonctionnement versée au gestionnaire d'établissement qui accueille des enfants âgés de moins de 6 ans. La Psu est égale à $66 \%$ du prix de revient horaire de l'accueil de l'enfant dans la limite du prix plafond fixé annuellement par la Cnaf, déduction faite des participations familiales. Depuis 2014, le montant de la prestation est modulé en fonction du service rendu. Afin de bénéficier de ce soutien, l'établissement doit calculer les participations familiales à partir d'un barème national fixé par la Cnaf. Ce barème s'applique aux revenus du ménage en tenant compte du nombre d'enfants à charge.

Mise en œuvre en 2002 (revue en 2003, 2011, 2014 et 2019), la Psu répond à la nécessité de rendre accessibles les Eaje à de nouveaux publics. L'objectif est de permettre à un plus grand nombre d'enfants de bénéficier d'une place en crèche, d'ouvrir les Eaje à un public mixte, d'optimiser les places d'accueil existantes en augmentant leur taux d'occupation. Pour y avoir droit, les gestionnaires doivent s'engager à appliquer le barème de tarification de la Cnaf qui permet de calculer un tarif modulé en fonction des ressources des familles et de leur composition et à une contractualisation établie à partir des besoins réels exprimés par ces familles.

(Source : Lettre Circulaire Cnaf Psu 2011-105 du 29 juin 2011).

${ }^{7}$ Cette ouverture à de nouveaux publics a été réaffirmée plus récemment dans le cadre de la stratégie de lutte contre la pauvreté (encadré 3, p. 33).

Revue des politiques sociales et familiales $n^{\circ} 136-137-3^{e}$ et $4^{e}$ trimestres 2020

32 Socialisation des enfants - Intentions, attentions et injonctions 
risco ». " Ton groupe là, il y a combien de périsco ? » (auxiliaire à l'adresse d'une collègue qui s'apprête à conduire un groupe d'enfants dans la salle du repas, crèche associative).

La stratégie de prévention

et de lutte contre la pauvreté

Plus communément désignée sous le terme « plan pauvreté », la stratégie de prévention et de lutte contre la pauvreté a été présentée par le gouvernement en septembre 2018. Concernant la petite enfance, parmi ses objectifs, elle prévoit de développer des solutions d'accueil collectif des jeunes enfants en mettant en place des incitations financières en fonction de la localisation des places d'accueil afin de permettre une réduction des coûts, notamment en zone prioritaire, et une plus grande mixité sociale au sein des établissements d'accueil du jeune enfant (Eaje).

Pour faciliter l'accueil d'enfants issus de familles précaires, le bonus " mixité sociale » a été mis en place pour compenser le manque de recettes qui pourrait apparaître pour les gestionnaires, notamment en raison de temps $d^{\prime}$ accueil plus courts en moyenne pour ces enfants. Afin de répondre aux besoins de places de crèches, au moins trente mille places supplémentaires seront créées d'ici à 2022. Pour répondre à ces attentes, l'État a mis en place, par l'intermédiaire de la convention d'objectifs et de gestion (Cog) signée avec la Caisse nationale des Allocations familiales, des incitations financières en fonction de la localisation des places d'accueil tel que le bonus « territoires ". Il permet de réduire le reste à charge pour les communes les plus pauvres et donc de réduire le coût par place de crèche à moins de 1500 euros tout en rétablissant une certaine équité territoriale.

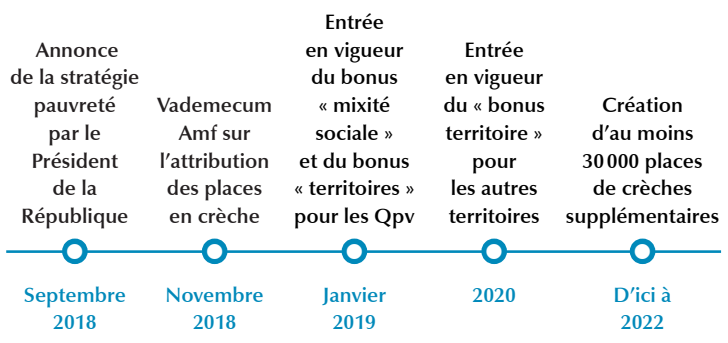

Voir la présentation de la stratégie sur le site du ministère

des Solidarités et de la Santé : https://solidarites-sante.gouv.fr/IMG/ pdf/strategie_pauvrete_vfhd.pdf (consulté 10 avril 2020).

Amf : Association des maires de France ; Qpv : quartier prioritaire de la ville.

Loin d'être neutres, ces pratiques langagières sont indissociables des relations sociales qui s'effectuent à travers elles (Lahire, 1992) et ces désignations d'enfants semblent en résonnance avec des conceptions éducatives apprises en formation et transmises dans les structures. La dimension axiologique de ces trois catégories met en lumière la manière dont ces différentes modalités d'inscription à la crèche peuvent être en écho ou en tension avec les conceptions du travail.
Les « réguliers » qui catégorisent les enfants inscrits selon un contrat établi sur la base d'un nombre d'heures mensuelles, quel que soit ce nombre d'heures, renvoient, dans le domaine éducatif, à un certain type d'enfant : ceux qui se conforment facilement aux rythmes de la crèche, qui prennent leurs repas et se reposent à des heures régulières. " Avec les bébés, au début, on demande aux parents de remplir le cahier de rythmes, comme ça on sait quand il a mangé et on peut mieux voir son rythme, s'il est régulier ou si c'est encore chaotique... »(Aux, crèche municipale). La directrice d'une crèche municipale explique en présentant sa structure : "On a de plus en plus de parents qui ont peur de mettre un cadre. Les enfants sont levés, couchés à n'importe quelle heure [...]. Notre travail c'est de les aider à installer de la régularité. On leur explique que, pour leur santé et pour l'école après, il leur faut des horaires réguliers... » (directrice, crèche municipale). La catégorie des « réguliers » créée par différence avec les deux autres catégories, semble faire référence à des attendus sociaux et professionnels en adéquation pour les professionnelles, avec les apprentissages de la formation. Même si les enfants "réguliers » sont bien différents de ces représentations, cette désignation semble les inclure dans un cadre d'action cohérent où les professionnelles se sentent à l'aise.

La catégorie « occasionnel » opère à l'inverse. Synonyme dans le dictionnaire de " contingent», $\mathrm{d}^{\prime}$ " accidentel» ou $d^{\prime}$ " intermittent», ce terme est en rupture avec la régularité prônée dans le champ éducatif. Les " occasionnels "sont accueillis lorsque des places sont laissées vacantes par "les réguliers », parce que ces derniers sont malades, en vacances, ou lors de journées moins fréquentées, tels les mercredis. Les directrices appellent les parents inscrits dans ce cadre, souvent le jour même lorsqu'un enfant malade laisse vacante sa place. Sur une même place peuvent être accueillis successivement plusieurs enfants à différents moments de la journée ou jours de la semaine. "Avec la directrice, le matin, on pointe les absences et on appelle les occasionnels... quel que soit le moment dans l'année, on a toujours autant d'enfants » (Eje, crèche associative).

Ce type d'accueil vient non seulement empêcher ou gêner la régularité souhaitée pour l'éducation d'un enfant mais contrarie aussi l'anticipation qui permet à la professionnelle de se projeter dans sa journée : "Les occasionnels, c'est parfois compliqué pour eux de s'intégrer aux groupes. Ils viennent peu et l'équipe apprend souvent le matin même que l'enfant sera présent, parce qu'une place est libre »

Revue des politiques sociales et familiales $n^{\circ} 136-137-3^{e}$ et $4^{e}$ trimestres 2020 
(directrice crèche associative). Cette désignation contraint à prendre en compte la contingence, qui est une notion rarement employée par les professionnelles et peu développée en formation, même si les observations tendent à montrer que les professionnelles ne cessent de s'ajuster à l'imprévisibilité des enfants. D'une certaine manière contraire à une éducation structurante, ce principe d'accueil occasionnel vient heurter la régularité à privilégier.

Enfin, la catégorie "périscolaire », dite " périsco », renvoyant à l'univers scolaire, paraît ne pas faire sens, tant les professionnelles travaillant dans les crèches semblent avoir intégré la séparation institutionnelle entre les ministères de la santé et de l'Éducation nationale pour la prise en charge des tout-petits. Le préfixe grec "peri- », signifiant " autour » montre bien que ces enfants ne sont pas au centre, mais à part. Pour les professionnelles rencontrées, la catégorie des "périsco » est hors de leurs préoccupations et à éviter. "Pour l'instant, des périsco, on n'en a pas beaucoup, mais la directrice nous a prévenus, à la rentrée on n'y coupe pas, elle est obligée d'appliquer les directives ! »(Eje, crèche municipale). S'attacher à rendre les enfants "prêts pour l'école » et les voir revenir à la crèche, semble soit « incompréhensible », soit dégradant : " avec les périsco, pour moi, on quitte I'accueil et on revient à la garde. On les garde, c'est tout » (Aux., crèche municipale). "C'est paradoxal, on les a bassinés depuis la fin de leur dernière année en crèche en leur disant "tu es grand, tu vas aller à l'école en septembre" et là ils reviennent à la crèche...Quel sens ça a pour l'enfant? "(Aux., crèche associative).

Ces deux catégories en tension avec des conceptions éducatives portées implicitement par les professionnelles, font souvent l'objet de longs développements quand les chercheurs échangent avec elles à ce propos. Peut-être ces points de vue en disent-ils moins sur le rapport à ces enfants et leur prise en charge que sur les difficultés qu'elles éprouvent par rapport à l'augmentation de la charge de travail et la capacité d'adaptation dont elles doivent faire preuve? "On est entrés dans un système économique qui fait qu'on est obligés de faire $d u$ chiffre, on n'a pas de marge de manœuvre. Ça impacte notre travail éducatif. II n'y a jamais de moment où les effectifs sont plus bas, où on est tranquille. Dès qu'il y a une absence, la directrice appelle pour remplir avec les occasionnels, mais l'équipe n'apprécie pas, car elles perdent en qualité de travail. C'est un problème, car certains enfants ont du mal à s'intégrer dans le groupe [...], ce n'est pas rassurant pour eux. » (Eje en codirection).

\section{Disjonction des manières de dire et des manières de faire}

Ces trois catégories d'enfants et les longs développements des différentes professionnelles laissaient supposer des différences dans la manière de prendre en charge ces enfants. Les observations montrent des écarts entre les manières de dire et de faire. Les enfants " occasionnels et périsco » ne bénéficient ni ne pâtissent de prises en charge vraiment différentes des autres. Ainsi, au début de la recherche, alors que les observations portaient sur une appréhension globale de la structure et des groupes, rien ne permettait de distinguer, par rapport à leur prise en charge, ces enfants des autres. C'est après plusieurs semaines d'observation et d'écoute attentive du vocabulaire mobilisé par les professionnelles, et à la suite d'entretiens, que sont apparues ces catégories et les différences qu'elles pouvaient engendrer.

Un premier étonnement à propos des "périsco " a été de constater que, contrairement, à un alourdissement de la tâche, ils pouvaient la faciliter, notamment pour gérer le groupe d'enfants "Tu es grand, toi, tu vas à l'école, ne fais pas le bébé et mange proprement! » (Aux., crèche municipale). Pourtant, les professionnelles n'ont pas réellement conscience de ces facilités permettant $d$ 'inciter l'enfant à plus d'autonomie. De la même façon, à une remarque émise par l'observateur un jour à une auxiliaire de puériculture sur la sérénité de sa tablée au cours du repas qui s'est déroulé, et à une question sur la composition du groupe d'enfants, elle s'exclame presque surprise : "C'est vrai, c'est marrant, il n'y a presque que des périsco [cinq sur sept], je n'avais pas fait attention!". Ainsi, au lieu de souligner l'opportunité de mobiliser ces enfants pour gérer plus facilement leur groupe, ces professionnelles semblent focalisées sur la pertinence de leur présence au sein de la structure. Pour les observateurs, les épreuves pour maîtriser la complexité paraissent moindre avec ces enfants, mais un effet de halo maintient, pour les professionnelles, la perception d'une charge de travail accrue. Hors champ des conceptions pédagogiques, le soutien de ces "périsco » pour la gestion d'un groupe ne semble pas être pris en compte dans les ajustements minutieux effectués pour la répartition des charges de travail au sein des équipes.

Ces catégorisations paraissent engendrer un décryptage différent du comportement des enfants. Cette difficulté semble liée à la nouveauté de certaines situations sociales que les professionnelles ne sont pas habituées à prendre en charge. Une auxiliaire de puériculture

Revue des politiques sociales et familiales $n^{\circ} 136-137-3^{e}$ et $4^{e}$ trimestres 2020 
souligne ainsi la difficulté de la relation avec certaines familles pour qui l'accueil en crèche est une incitation des services sociaux et non un choix éducatif. "Pour certains parents, cela ne fait pas sens de mettre un enfant en crèche. Ils l'emmènent tard le matin, viennent le chercher un peu n'importe quand... Parfois, on ne voit pas l'enfant pendant trois semaines, puis il revient sans explication... c'est difficile car il faut toujours qu'il se réadapte au collectif et nous, à lui » (Aux., crèche municipale).

À propos d'un petit garçon qui refuse de participer à une activité avec le groupe, une professionnelle souligne : "Évidemment, Yoann, ça fait presque un mois qu'il n'est pas venu! »(Cap, crèche associative). Les pleurs des enfants « occasionnels » peuvent être interprétés à l'aune de leur fréquentation irrégulière de la crèche là où le même comportement chez un enfant régulier ne posera pas de question. "Elle se roule encore par terre, c'est très difficile pour elle de se séparer... En même temps, elle vient tellement peu souvent! » explique une Eje en crèche associative à propos du comportement d'une petite fille en accueil occasionnel après le départ de sa mère. Les pleurs, la difficulté qu'un enfant "régulier » va avoir à se séparer de ses parents sera envisagé différemment, par exemple à l'aune de son caractère ou de la similitude avec celui de ses frères et ses sœurs : Tom est un petit garçon âgé de 18 mois qui a commencé son adaptation il y a trois semaines. II vient trois demi-journées chaque début de semaine et ne prend pas encore ses repas à la crèche. Dès que sa mère s'en va, il s'agrippe à elle, pleure et reste une partie de la matinée en retrait isolé du groupe avec son doudou. Dans le cas de Tom, les difficultés de séparation de l'enfant s'inscrivent dans un processus $\mathrm{d}^{\prime}$ adaptation progressive auquel les professionnelles sont habituées. Tom, d'une certaine manière, vient illustrer et valider la place des savoirs appris en formation sur les théories de I'attachement (Neyrand, 2011), qui confirment l'importance d'un protocole d'adaptation progressive, que les professionnelles mettent en place avec le souci de réussir cette séparation avec les parents.

La présence irrégulière de ces « occasionnels » modifie nécessairement ces protocoles et ébranlent les usages de ces savoirs dans les pratiques professionnelles. Comment les professionnelles peuvent-elles concilier leurs apprentissages relatifs à la « souffrance de la séparation », "la continuité pédagogique » ou « I'adaptation progressive » avec ces situations nouvelles où le temps de présence à la crèche s'appréhende différemment ? Comment concilier les pratiques de travail avec les évolutions des politiques sur l'accueil des tout-petits quand les normes qui fondent les pratiques professionnelles ne font pas aussi l'objet de questionnements ? Dans ces différentes situations, les professionnelles sont soucieuses de la qualité de la prise en charge des enfants. Qu'ils soient "réguliers », " occasionnels » et même "périscos », elles s'attachent à répondre à leurs demandes. Cependant, l'interprétation des mobiles du comportement des enfants n'étant pas la même, la manière de s'engager dans l'action pour répondre diffère nécessairement. À propos d'un groupe d'enfants qui joue un peu à part, une auxiliaire de crèche associative souligne "oui, mais là, ce sont des périsco ». Cette remarque renseigne sur la place particulière qui est celle de ces enfants dans la représentation et I'appréhension qu'elle en a. Elle questionne le développement des compétences permettant d'accompagner les professionnelles à prendre en compte les transformations du travail, notamment celles liées aux évolutions des politiques publiques.

\section{Conclusion}

Cette recherche sur les catégorisations mobilisées dans l'action a mis en évidence les complexités de ce processus qui ne peut se saisir par la seule observation mais requiert la mobilisation de plusieurs cadres conceptuels complémentaires, notamment sociolinguistique et phénoménologique. Par I'attention au langage mobilisé dans l'action, I'analyse montre que le processus de catégorisation constitue une ressource pour ajuster équitablement dans l'action les charges de travail au sein des équipes, jauger des compétences de collègues en fonction de critères subtils et concilier attentes parentales, normes et valeurs professionnelles.

Combinant les connaissances et les expériences, le regard porté sur l'enfant est construit à partir de plusieurs prismes qui articulent, juxtaposent ou mettent en tension une pluralité de normes apprises, transmises, imposées ou construites, toujours recomposées dans le cours de l'action. Dès lors, les difficultés à circonscrire la professionnalité de ces salariées sont évidentes. Cette professionnalité paraît relever d'une construction singulière, faite de "bric et de broc », pour reprendre les termes de Jean-Blaise Grize (1998), dont la spécificité semble justement tenir à cette hétérogénéité qui se reconfigure en permanence en fonction des organisations du travail, des activités conduites et des enfants accueillis.

Revue des politiques sociales et familiales $n^{\circ} 136-137-3^{e}$ et $4^{e}$ trimestres 2020

35 Socialisation des enfants - Intentions, attentions et injonctions 
Si ce processus de catégorisation constitue une ressource essentielle de l'action, la recherche montre également des tensions entre les évolutions sociales nécessitant pour les crèches de diversifier leurs offres d'accueil et les formations dispensées. Ces savoirs appris en formation paraissent en tension avec le service aujourd'hui attendu des Eaje concernant l'accueil d'une plus grande diversité de publics. La référence à certaines normes, cadres de référence, théories qui sont donnés comme stables et intangibles, est questionnée par les évolutions du travail. En s'attachant à faire tomber «l'écran que les mots placent devant nous »(Becker, 2002), les termes de « occasionnel », "périscolaire » et "régulier», apparemment neutres, sont ainsi en étroite filiation avec ces conceptions éducatives qui déterminent un certain rapport aux enfants. L'analyse des catégories créées par les professionnelles invite à rester au plus près des pratiques pour comprendre les oscillations toujours possibles entre la stigmatisation et la nonstigmatisation, en fonction de la complexité de certains contextes. Les crèches où les observations ont eu lieu sont des structures où les possibilités de réflexions collectives et de régulation existent pour échanger et travailler les situations difficiles, qu'elles soient liées ou non à ces orientations politiques. Qu'en est-il de I'usage de ces catégories dans les établissements où les professionnelles ne bénéficient pas de ces instances collectives de travail ?

Arasse D., 2003, On n'y voit rien. Descriptions, Folio Essais, Paris, Gallimard.

Barbier J.-M., 2011, Vocabulaire d'analyse des activités. Formations et pratiques professionnelles, Paris, Presses universitaires de France.

Becker H., 2002, Les ficelles de métier, Paris, La Découverte.

Boltanski L., 1969, Prime éducation et morale de classe, Cahier du Centre de sociologie européenne, Évreux, Mouton.

Borzeix A., Fraenkel B. (dir.), 2001, Langage et travail, Communication, cognition, action, Paris, Cnrs Éditions. Bourdieu P., 1993, La misère du monde, Paris, Seuil.

Cartier M., Lechien M. H., Meuret Campfort E., 2014, "Introduction », Hiérarchies et conflictualité dans I'accueil des petits enfants, Sociétés contemporaines, $n^{\circ}$ 95, p. 5-28.

Clot Y., 2007, De I'analyse des pratiques au développement des métiers, Éducation \& Didactique, $n^{\circ} 1$, p. 83-93.

Cresson G., 1998, Formations et compétences dans les métiers de contact direct avec les petits enfants : quelques enjeux, conflits et paradoxes, Lien social et Politiques, n 40, p. 25-37.

Garfinkel H., 2007[1967], Recherches en ethnométhodologie, Paris, Presses universitaires de France Quadrige.

Giampino S., 2016, Développement du jeune enfant-Modes d'accueil, formation des professionnels, rapport pour le ministère des Familles de l'Enfance et des Droits des femmes, https://solidarites-sante.gouv.fr/IMG/pdf/ rapport-giampino-vf_modif-17_08_16.pdf (consulté le 8 avril 2020).

Goffman I., 1973, La mise en scène de la vie quotidienne, Paris, Éditions de Minuit.

Goodwin, C., 1994, Professional vision. American Anthropologist, vol. 96, n 3, p. 606-633.

Grize J.-B., 1998, Savoirs théoriques et savoirs d'action : point de vue logico-discursif, in Barbier J.-M., (dir), Savoirs théoriques et savoirs d'action, Paris, Presses universitaires de France.

Haut Conseil de la famille, de l'enfance et de l'adolescence (Hcfea), 2019, Pilotage de la qualité affective, 
éducative et sociale de l'accueil du jeune enfant - Rapport adopté par consensus par le Conseil de l'enfance et de l'adolescence le 22 mars 2019, site https://www.strategie.gouv.fr/sites/strategie.gouv.fr/files/atoms/files/ hcfea-rapport-qualite-affective-avril-2019.pdf (consulté le 25 mai 2020).

Houdé O., 1992, Catégorisation et développement cognitif, Paris, Presses universitaires de France.

Hurtig M.-H., 2019, La Psu 2014, des effets paradoxaux et un risque de perte de sens ?, in Moisset P. (dir.), Accueillir la petite enfance : le vécu des professionnels, Toulouse, Érès, p. 75-100.

Jobert G., 2019, La formation professionnelle comme éducation du regard au travail, Ergologia, décembre, $\mathrm{n}^{\circ} 22$, p.23-49.

Lacoste M., 1999, Communication et intelligence collective. Le travail à l'hôpital, Paris, Presses universitaires de France.

Lahire B., 1992, Précisions sur la manière sociologique de traiter du " sens » : quelques remarques concernant l'ethnométhodologie, Langage et société, n 59, p. 73-89.

Le Floch M.-C., 2008, Une relecture du sale boulot. Entre une division morale et une division sociale du travail éducatif, Pensée plurielle, $n^{\circ} 18$, p. 31-48.

Ministère des Solidarités et de la Santé (Mss), 2018, Stratégie nationale de prévention et de lutte contre la pauvreté, https://solidarites-sante.gouv.fr/IMG/pdf/strategie_pauvrete_vfhd.pdf (consultation le 10 avril 2020).

Neyrand G., 2011, L'enfant, la mère et la question du père. Un bilan critique de l'évolution des savoirs sur la petite enfance, Paris, Presses universitaires de France.

Odena S., 2019, Organisation du travail dans les crèches, conditions de travail, modalités d'exercice des professions, in Moisset P. (dir.) Accueillir la petite enfance : le vécu des professionnels, Toulouse, Érès, p.157-174.

Odena S., 2012, Travailler en crèche : un choix par défaut et une hétérogénéité source de tensions, Politiques sociales et familiales, $\mathrm{n}^{\circ} 109$, p. 23-33.

Odena S., 2009, Les professions et leur coordination dans les établissements d'accueil collectifs du jeune enfant : une hétérogénéité source de tensions au sein des équipes, Dossiers d'études, Caisse nationale des Allocations familiales, $\mathrm{n}^{\circ} 121$.

Olivier de Sardan J.-P., 1995, La politique de terrain. Sur la production des données en anthropologie, Enquête - Les terrains de l'enquête, https://sspsd.u-strasbg.fr/IMG/pdf/Olivier_de_Sardan_1995_enquete-2631-la-politique-du-terrain.pdf (consulté le 3 juin 2020).

Rayna S., 2003, Regards sur les apprentissages des tout-petits, Enfances \& Psy, p. 30-41.

Schutz A., 2008[1987], Le chercheur et le quotidien, Paris, Klincksiek.

Schwartz O., 2011 [1993], L'empirisme irréductible. La fin de l'empirisme ?, in Anderson N. (dir.), Le Hobo, sociologie du sans-abri, Paris, Armand Colin, p. 336-380.

Ulmann A.-L., Betton, E., Jobert G., 2011, L'activité des professionnelles de la petite enfance, Dossiers d'études, Caisse nationale des Allocations familiales, $\mathrm{n}^{\circ} 145$.

Ulmann A.-L., Rodriguez D., Guyon, M., 2015, Former les futurs professionnels de la petite enfance. Entre soins et éducation, quelle place pour les affects?, Politiques sociales et familiales, $n^{\circ} 120$, p. 29-41.

Revue des politiques sociales et familiales $n^{\circ} 136-137-3^{e}$ et $4^{e}$ trimestres 2020

37 Socialisation des enfants - Intentions, attentions et injonctions 